\title{
Thirty years of standalone percutaneous coronary interventions: A 23,261 case experience from a Canadian tertiary referral centre
}

\author{
William KK Hui MBBS FRCP(Edin) FRCPC FACC FHKAM FHKCP ${ }^{1}$, David G O'Brien MBChB MD FRCP(Lond) ${ }^{1}$, \\ Neil S Brass MD FRCPC FSCAI ${ }^{1}$, Po Kee Cheung MBBS FRCPC ${ }^{1}$, Michael CY Chan MBBS FRCPC FACC ${ }^{1}$, \\ Raymond CM Leung MD FRCPC ${ }^{1}$, Benjamin D Tyrrell MD FRCPC ${ }^{1}$, Shantu Bundhoo MBChB MD MRCP(UK) ${ }^{1}$, \\ Pitak Pongnonthachai MD¹, P Diane Galbraith BN MSc², Keysun Ranjbar MD FESC ${ }^{1}$, \\ Micha Dorsch MD PhD MRCP(UK $)^{1}$, Roderick MacArthur MD FRCSC ${ }^{3}$, W Peter Klinke MD FRCPC
}

\begin{abstract}
WKK Hui, DG O'Brien, NS Brass, et al. Thirty years of standalone percutaneous coronary interventions: A 23,261 case experience from a Canadian tertiary referral centre. Curr Res Cardiol 2015;2(4):183-187.
\end{abstract}

BACKGROUND: When percutaneous coronary intervention (PCI) was first performed $>35$ years ago, on-site cardiac surgery backup for PCI failure was deemed essential. As techniques improved and primary PCI became the preferred reperfusion strategy for acute myocardial infarction, many standalone PCI programs originally established to facilitate access to primary PCI started to perform elective PCI, which remains a Class IIb recommendation in American College of Cardiology Foundation/American Heart Association/Society for Cardiac Angiography and Interventions guidelines. Two recent United States studies showed that outcomes of elective PCI in standalone centres were noninferior to those with on-site cardiac surgery. The Royal Alexandra Hospital in Edmonton (Alberta) performed the first standalone PCI in Canada in 1981.

OBJECTIVES: The authors describe their first 30 years' experience with $\mathrm{PCI}$ - the largest single-centre standalone experience reported to date.

Dercutaneous coronary intervention (PCI) is currently the predominant form of coronary revascularization. In the United States, $63.3 \%$ of 553,159 coronary revascularization procedures in 2009 were percutaneous interventions (1). In stable coronary disease, PCI improves symptoms of angina (2,3). In acute coronary syndromes, timely restoration of normal coronary flow improves survival $(4,5)$. Primary PCI has become the treatment of choice for acute ST elevation myocardial infarction (STEMI) (6,7). Better outcomes with shorter call-to-balloon times have prompted a significant number of 'diagnostic angiography only' centres to commence PCI programs without on-site cardiac surgery backup, ie, standalone PCI programs (8). Although standalone PCI has many advantages in terms of logistic and economic considerations, some concerns remain as to the safety of such practice $(9-12)$.

Nonetheless, new standalone PCI programs continue to be established worldwide with high procedural success and no apparent excess mortality or need for emergency coronary artery bypass grafting $(\mathrm{CABG})$ compared with centres with on-site surgery (13-20). The latest American College of Cardiology Foundation (ACCF)/American Heart Association (AHA)/Society for Cardiac Angiography and Interventions (SCAI) Guideline on Percutaneous Coronary Intervention, published in 2011 (21), supported and upgraded
METHODS: Patient and procedural data have been collected since the first standalone PCI in 1981, evolving from paper records to a computer database and, in 1995, a provincial database. Quality assurance and peer review in collaboration with the regional cardiac surgery program was established from the outset.

RESULTS: The success, emergency coronary bypass and death rates for 23,261 standalone PCIs performed between 1981 and 2011 were $96.0 \%$, $0.2 \%$ and $0.5 \%$, respectively. For 9068 PCIs performed between 2007 and 2011 (a period that most reflects contemporary practice), the rates were $96.5 \%, 0.04 \%$ and $0.6 \%$, respectively. These results compare favourably with PCI results reported in literature, irrespective of the presence or absence of on-site cardiac surgery.

CONCLUSIONS: With high volumes and an experienced team, standalone PCIs can be performed safely with excellent success and low complication rates.

Key Words: Cardiac surgery backup; Coronary revascularization; Standalone percutaneous coronary intervention

standalone PCI for STEMI from Class IIb to Class IIa recommendation. However, caution remained with regard to elective PCI, despite having also been upgraded from Class III to Class IIb recommendation (21). More recently, Canadian researchers performed a meta-analysis of $>100,000$ primary and $>900,000$ nonprimary PCIs, comparing outcomes in standalone PCI centres versus those with on-site cardiac surgery, and found no increased risk for in-hospital mortality or early coronary bypass rates in standalone PCI centres (22). The US Cardiovascular Patient Outcomes Research Team (CPORT-E) (23) and Percutaneous Coronary Intervention (PCI) Outcomes in Community Versus Tertiary Settings (MASS COMM) (24) trials also showed that outcomes of nonemergency PCIs performed in hospitals without on-site cardiac surgery were noninferior to that performed in hospitals with on-site surgery, although highrisk patients were excluded in both trials.

The Royal Alexandra Hospital in Edmonton, Alberta, performed the first standalone coronary angioplasty in Canada in July 1981. Our first 10 years of standalone PCI experience in 762 patients has previously been reported (25). In the present article, we describe our first 30 years' experience with 23,261 cases, which we believe is the largest single-centre, standalone PCI experience reported to date.

${ }^{1}$ CK Hui Heart Centre, Royal Alexandra Hospital; ${ }^{2}$ Alberta Provincial Project for Outcomes Assessment in Coronary Heart Disease (APPROACH);

${ }^{3}$ Division of Cardiac Surgery, Mazankowski Alberta Heart Institute, Edmonton, Alberta

Correspondence: Dr William KK Hui, 6th Floor, C K Hui Heart Centre, Robbins Pavilion, Royal Alexandra Hospital, 10240 Kingsway Avenue,

Edmonton, Alberta T5H 3V9. Telephone 780-735-5962, fax 780-735-6833, e-mail william@huico.com 
TABLE 1

Royal Alexandra Hospital (Edmonton, Alberta) percutaneous coronary intervention (PCI) results, 1981 to 2011

\begin{tabular}{|c|c|c|c|c|c|}
\hline & 1981-1991 & $1992-2001$ & $2002-2006$ & $2007-2011$ & 30 years \\
\hline $\mathrm{PCl}, \mathrm{n}$ & 762 & 5909 & 7522 & 9068 & 23,261 \\
\hline Ad hoc PCls, \% & $\mathrm{n} / \mathrm{a}$ & 62.6 & 75.4 & 85.5 & 76.1 \\
\hline Canadian Cardivascular Society class IV, \% & $\mathrm{n} / \mathrm{a}$ & 60.8 & 67.0 & 69.1 & 66.2 \\
\hline Primary/rescue $\mathrm{PCl}, \%$ & $\mathrm{n} / \mathrm{a}$ & 7.0 & 18.1 & 15.2 & 14.0 \\
\hline Stent use (since 1993), \% & $\mathrm{n} / \mathrm{a}$ & 70.4 & 91.3 & 93.0 & 82.3 \\
\hline Procedural success, $\%$ & 73.1 & 94.8 & 96.2 & 96.5 & 96.0 \\
\hline
\end{tabular}

CABG Coronary artery bypass grafting; $n / a$ Data not available

\section{METHODS}

\section{PCI program structure}

The Royal Alexandra Hospital is one of two tertiary care hospitals in Edmonton, with $>900$ beds. It is a major referral centre for patients from central and northern Alberta, adjacent provinces and the Northwest Territories beyond the Arctic circle. Regional and interprovincial patient services utilize ground ambulance, helicopters as well as fixedwing air ambulance for transport of patients to the facility. A cardiac catheterization and angiography program was set up in collaboration with diagnostic imaging in the 1960s. In the late 1970s, the first cardiac catheter laboratory was established and a second laboratory was added in 1999. A new heart centre with state-of-the-art facilities and equipment opened in 2011.

The centre was one of the first to demonstrate the safety of outpatient cardiac catheterizations (26) and, in Canada, one of the first to adopt coronary angioplasty as a technique for coronary revascularization early in its development, supported by the regional cardiac surgery program at the University of Alberta Hospital $6 \mathrm{~km}$ away (approximately $15 \mathrm{~min}$ door-to-door), with an operating room (OR) on standby while the procedure was performed at the site. Due diligence with the transfer process for PCI patients who required urgent CABG, including pilot runs to confirm logistics and feasibility of patient transport with intra-aortic balloon support, were performed before the first coronary angioplasty in July 1981.

Balloon angioplasty was the only PCI technique available in the first 12 years of the program. The first coronary stent was implanted in 1993. Glycoprotein IIb/IIIa inhibitors were introduced in 1997 and drug eluting stents in 2002. A radial PCI program started in 1999 and is now used in $>80 \%$ of cases. Ancillary interventional techniques, such as intravascular ultrasound, fractional flow reserve, thrombectomy/distal protection and rotational atherectomy have all become an integral part of the program. Intra-aortic balloon pump has been available since 1984 and Impella (Abiomed, USA) circulatory support since 2008. A mitral valvuloplasty program was started in 1988 and septal ablation program for hypertrophic cardiomyopathy in 2005. A chronic total occlusion PCI program was started in 2010. An interventional cardiology fellowship program was established in 1991 and has trained $>20$ cardiologists from Australia, England, China, Hong Kong, Netherlands, Saudi Arabia, Singapore, Sweden, Thailand and Wales.

Initially, all potential PCI cases were reviewed weekly at a meeting attended by interventional cardiologists and cardiac surgeons. By the 1990s, as PCI volumes grew and more ad hoc PCIs were performed, separate weekly PCI and surgical case review meetings were held. PCI review included all emergency cases, complications and choice of revascularization and $\mathrm{PCI}$ approach in complex cases. As case volumes increased, uncomplicated emergency cases were not reviewed. Weekly meetings with cardiac surgeons included review of surgical cases as well as cases suitable for either percutaneous or surgical approach. Surgical backup was initially provided on an OR standby basis and, subsequently, on a next available OR basis since the early 1990s.
Data collection

Since the start of the PCI program in July 1981, comprehensive data regarding indication, procedural success, complications, mortality and need for emergency CABG surgery were collected. In the first 10 years, only paper records were kept and archived for quality assurance. Since 1991, records have been kept in dBASE format and, from 1995 onward, these were kept in parallel with a new provincial database Alberta Provincial Project for Outcomes Assessment in Coronary Heart Disease (APPROACH) (27) until 2002. Since 2002, all records have been kept exclusively in the APPROACH database.

Medical records for all cases requiring cardiac surgery within $24 \mathrm{~h}$ of PCI were reviewed to confirm the timing of surgery, indication and operative outcomes. All deaths within $24 \mathrm{~h}$ of PCI, including all primary/rescue PCIs and patients in cardiogenic shock, were crossreferenced with hospital and regional health records. Patient survival was tracked by the APPROACH database through a linkage to provincial vital statistics.

\section{RESULTS}

From 1981 to 2011, 23,261 PCIs were performed. The results of the standalone PCI experience over four time periods, 1981 to 1991, 1992 to 2001, 2002 to 2006, and 2007 to 2011, are shown in Table 1.

Volumes of ad-hoc PCI and primary/rescue PCI for STEMI steadily increased. The last five-year period has had the greatest percentage of patients with Canadian Cardiovascular Society (CCS) Class IV angina. Lesion complexity was high. An annual audit in 2002 revealed that of 1573 lesions treated, 1098 (70\%) were anatomically complex, classified as ACC/AHA B2 or C (10). This compared with $58.2 \%$ in 1996 and $18.6 \%$ in 1990 to 1991.

The PCI volume has exceeded 1100 per year since 2000 and 1500 per year since 2004 . In parallel, the interventional cardiologist average case volume also increased from 170 PCIs per year in 1992 to 1996, to $\geq 250$ PCIs per year since 2002 .

The period from 2007 to 2011 most reflected contemporary practice and represented almost $40 \%$ of the total PCIs performed over the 30-year period. Of 9068 PCI cases performed, 1384 (15.3\%) were primary/ rescue PCI for acute myocardial infarction, of which 354 (3.9\%) were hemodynamically unstable. There were 4857 (53.6\%) urgent inpatients with acute coronary syndrome and 2473 (27.3\%) urgent outpatient cases. Only 354 (3.9\%) cases were classified as low risk.

Procedural success was achieved in $96.5 \%$. PCI was performed on an ad hoc basis in $85.5 \%$ of cases. Emergency CABG surgery was required in four patients $(0.04 \%)$, three due to PCI failure and, in one unstable patient, PCI was performed on a severe left main lesion as a bridge to surgery after discussion with the cardiac surgeon on-call. Mortality rate at $24 \mathrm{~h}$ was $0.6 \%$.

At 30 days, the mortality rate was $1.1 \%$. CABG was performed in $42(0.5 \%)$ patients and repeat PCI was performed in $510(5.6 \%)$, of whom $431(4.7 \%)$ were planned PCIs for nonculprit lesions and $79(0.9 \%)$ were unplanned - 39 for subacute stent thrombosis and 40 for residual nonculprit lesion ischemia. 
TABLE 2

Royal Alexandra Hospital (RAH, Edmonton, Alberta) percutaneous coronary intervention (PCI) results compared with benchmarks reported in the literature

\begin{tabular}{|c|c|c|c|c|c|}
\hline Study (reference) & Procedures, $\mathbf{n}$ & Primary $\mathrm{PCl}, \%$ & Lesion success, \% & Emergency CABG, \% & 30-day mortality, \% \\
\hline RAH (2007 to 2011) & 9068 & 15.3 & 96.5 & 0.04 & 1.1 \\
\hline \multicolumn{6}{|l|}{ Scotland (1997 to 2003) (41) } \\
\hline Low volume & 3756 & 8.2 & Not reported & 1.8 & 1.9 \\
\hline Medium volume & 10,419 & 2.9 & Not reported & 0.9 & 1.4 \\
\hline High volume & 3242 & 5.3 & Not reported & 0.4 & 1.4 \\
\hline \multicolumn{6}{|l|}{ Sweden (2000 to 2003) (19) } \\
\hline Standalone & 8838 & 18.0 & Not reported & 0.1 & 1.4 \\
\hline Surgery on-site & 25,525 & 9.7 & Not reported & 0.2 & 2.2 \\
\hline \multicolumn{6}{|l|}{ NCDR (2004 to 2006) (35) } \\
\hline Standalone & 8736 & 22.1 & 94 & 0.3 & $1.7^{*}$ \\
\hline Surgery on-site & 299,425 & 10.4 & 93 & 0.4 & $1.2^{\star}$ \\
\hline Tasmania (2005 to 2007) (20) & 1348 & 18.4 & 98 & 0.1 & $0.8^{*}$ \\
\hline \multicolumn{6}{|l|}{ CPORT-E (2006 to 2011) (23) } \\
\hline Standalone & 14,149 & Excluded & 93.4 & 0.1 & $0.9^{\dagger}$ \\
\hline Surgery on-site & 4718 & Excluded & 94.1 & 0.2 & $0.8^{\dagger}$ \\
\hline \multicolumn{6}{|l|}{ MASS COMM (2006 to 2011) (24) } \\
\hline Standalone & 2774 & Excluded & 95.6 & 0.3 & 0.7 \\
\hline Surgery on-site & 917 & Excluded & 97.1 & 0.1 & 0.3 \\
\hline
\end{tabular}

*In-hospital mortality; ${ }^{\dagger}$ Six-week mortality. CABG Coronary artery bypass grafting; CPORT-E Cardiovascular Patient Outcomes Research Team; MASS COMM Percutaneous Coronary Intervention (PCI) Outcomes in Community Versus Tertiary Settings; NCDR National Cardiovascular Data Registry

\section{DISCUSSION}

Since Andreas Gruntzig performed the first balloon coronary angioplasty in 1977 (28), the management of coronary disease changed forever. In the early years, acute coronary occlusion was the major complication limiting widespread application of this procedure. Our hospital had already performed coronary angioplasty for seven years by the time the first AHA/ACC coronary angioplasty guidelines were published in 1988, and subsequently updated in 1993, which recommended that angioplasty should not be performed without on-site cardiac surgery support $(9,10)$. Our first 10-year experience of standalone PCI (published in 1992 [24]) showed results comparable with then published benchmarks from centres with on-site cardiac surgery. However, our article was accompanied by two editorials, one of which questioned how our "approach ... will serve a useful role in interventional cardiology of the 1990s" (29), and the other concluded that "angioplasty should not be performed without on-site surgery facilities" (30).

Nonetheless, our OR standby for angioplasty in the 1980 s gave way to the "next available OR" model in the early 1990s because infrequent use made OR standby costly and impractical. Similar to experience reported elsewhere (31-33), coronary stents and adjunctive antiplatelet therapy greatly improved our success rate and reduced the incidence of death and emergency CABG in the 1990s.

In the past 15 years, reports from Israel (17), Norway (18), Sweden (19) and Tasmania (Australia) (20), as well as from rural and metropolitan centres in the United States (13-16), have shown that standalone PCI is safe and has comparable outcomes with centres with on-site cardiac surgery. A survey conducted by the SCAI in 2007 (8) reported that standalone $\mathrm{PCI}$ was practiced in $>90 \%$ of responding countries. The British Cardiovascular Intervention Society 2013 audit of PCI procedures (34) showed that standalone PCI centres have now significantly outnumbered those with on-site cardiac surgery (69 versus 48 ), with emergency CABG surgery rate $<1 \%$.

The marked improvement in the safety of coronary angioplasty, together with emerging evidence in support of primary PCI as the preferred treatment for STEMI, led to a change in the ACC/AHA guidelines for standalone primary PCI from a Class III recommendation in 1993 (10) to Class IIb in 2001 (11), and Class IIa in 2011 (21). In the United States, standalone PCI programs have become a necessity to provide primary PCI in rural areas (13), and to provide elective PCI in diagnostic catheter laboratories in metropolitan areas with experienced cardiologists and personnel from tertiary centres $(14-16)$. The very low risk of standalone PCI-related complications was considered to be justifiable, in the rural setting when balanced against potential delays in reperfusion in STEMI patients, and in the metropolitan setting against the costs as well as logistics involved in same-day transfer or second procedure scheduling for PCI in centres with on-site surgery. A 2009 report from the National Cardiovascular Data Registry (35) showed that standalone PCI centres had similar procedural success, morbidity, emergency surgery and risk-adjusted mortality rates compared with on-site surgery PCI centres, whether in primary PCI or less-urgent nonprimary settings. In 2011, the ACC/ AHA/SCAI guidelines standalone elective PCI was upgraded from a Class III (no benefit/harm) to a Class IIb (benefit $\geq$ risk, may be considered) recommendation. The safety of elective standalone PCI was further substantiated by CPORT-E (23) and MASS COMM (24) studies in 2012 and 2013, respectively.

Over the past 30 years, our program has built an experienced team of interventional cardiologists, nurses and technologists, with established protocols and quality assurance programs. It is well documented that higher operator and hospital volumes are associated with better patient outcomes (36-38). Our PCI volume rose from 762 cases in the first decade through 5909 cases in the second decade to 16,590 cases in the third decade of our experience. Our interventional cardiologist annual case exceeded both current United States and United Kingdom (UK) guidelines $(21,39)$. The broad experience of our interventional team is pivotal to success in complex cases, and to expedient and effective management of acute complications and emergencies. The comprehensive inventory of equipment that supports our case volumes also ensures that catheters, wires, balloons, stents and other new technologies essential for success are available when required. For 2007 to 2011, our procedural success, emergency CABG and death rates for a patient population of which $69.1 \%$ had CCS Class 4 symptoms and $15.2 \%$ required primary/rescue PCI, were comparable if not better than contemporary results reported worldwide $(19,20,23,24,35,40)$, some of which excluded emergency and primary PCI patients (Table 2).

In the past 10 years, percutaneous coronary revascularization has grown significantly in developed countries, while CABG surgery rates 
have remained static or declined. A 2010 report from the British Heart Foundation website (41) showed that PCI volumes in the UK increased by $350 \%$ from 1997 to 2008, whereas CABG rates peaked in 1997 and showed a flat to slight downward trend over the same period. Data from the United States showed that CABG rates declined 38\% from 2001 to 2008 (42). A 50\% decrease in CABG rate was also observed in Alberta from 2003 to 2010 (43).

In Canada, because of increasing demand for PCI services, a standalone PCI program was established as a pilot project by the Ontario provincial government at the Rouge Valley Hospital System in the Greater Toronto (Ontario) area in 2002. The program was closely monitored, and extensive procedural and outcome data collected. An expert panel report in 2004 (44) recommended continued government support of the program. Since then, five new standalone PCI programs have been established in Ontario: in Windsor and Thunder Bay in 2007, in Brampton and Peterborough in 2012, and in Niagara in 2014.

Despite these developments, with its vast land mass and significant distances between major cardiac centres in Canada, a recent study explored Canadian population access to primary PCI and found that only a small proportion of the country's geographical area was within 60 min of a PCI facility (45). Strategic placement of new PCI programs may improve such access.

In developing countries, as the incidence of coronary disease rise and new PCI centres are established, it does not appear logical nor cost effective to establish new cardiac surgery programs just to provide backup for the very rare instance that urgent $\mathrm{CABG}$ is required for PCI failure. However, a robust, fail-safe protocol for rapid transfer of

\section{REFERENCES}

1. Riley RF, Creighton WD, Powell W, Maynard C, Dean LS. Trends in coronary revascularization in the United States from 2001 to 2009. Cardiovasc Qual Outcomes 2011;4:193-7.

2. Parisi AF, Folland ED, Hartigan P. A comparison of angioplasty with medical therapy in the treatment of single-vessel coronary artery disease. N Engl J Med 1992;326:10-16.

3. Henderson RA, Pocock SJ, Clayton TC, et al. Seven-year outcome in the RITA-2 trial: Coronary angioplasty versus medical therapy. J Am Coll Cardiol 2003;42:1161-70.

4. Cannon CP, Weintraub WS, Demopoulos LA, et al. Comparison of early invasive and conservative strategies in patients with unstable coronary syndromes treated with the glycoprotein IIb/IIIa inhibitor tirofiban. N Engl J Med 2001;344:1879-87.

5. Mehta SR, Cannon CP, Fox KA, et al. Routine vs selective invasive strategies in patients with acute coronary syndromes: A collaborative meta-analysis of randomized trials. JAMA 2005;293:2908-17.

6. Antman EM, Anbe DT, Armstrong PW, et al. ACC/AHA guidelines for the management of patients with ST-elevation myocardial infarction - executive summary: A report of the American College of Cardiology/American Heart Association Task Force on Practice Guidelines (Writing Committee to Revise the 1999 Guidelines for the Management of Patients with Acute Myocardial Infarction). Circulation 2004; 110:588-636. (Erratum in 2005;111:2013).

7. Keeley EC, Boura JA, Grines CL. Primary angioplasty versus intravenous thrombolytic therapy for acute myocardial infarction: A quantitative review of 23 randomised trials. Lancet 2003;361:13-20.

8. Dehmer GJ, Blankenship J, Wharton TP Jr, et al. The current status and future direction of percutaneous coronary intervention without on-site surgical back-up: An expert consensus document from the Society for Cardiovascular Angiography and Interventions. Cathet Cardiovasc Intervent 2007;69:471-8.

9. ACC/AHA Task Force Report. Guidelines for percutaneous transluminal coronary angioplasty. J Am Coll Cardiol 1988;12:529-45.

10. Guidelines for percutaneous transluminal coronary angioplasty: A report of the American College of Cardiology/American Heart Association Task Force on Assessment of Diagnostic and Therapeutic Cardiovascular Procedures (Committee on Percutaneous Transluminal Coronary Angioplasty). J Am Coll Cardiol 1993;22:2033-54.

11. Smith SC Jr, Dove JT, Jacobs AK, et al. ACC/AHA guidelines of percutaneous coronary interventions (revision of the 1993 PTCA patients to backup surgical centres needs to be firmly established and tested. Regular case conference between interventional cardiologists and cardiovascular surgeons is invaluable not only for peer review, but also in developing the collegiality and trust that facilitate timely decisions and cooperation in emergency cases. Regulatory and professional organization guidelines for operator/institutional PCI volumes and quality assurance should be applied to all programs irrespective of the presence or absence of on-site cardiac surgery.

For years, the presence of on-site cardiac surgery has provided comfort to the cardiology community as a safety net for PCI programs. Institutions with cardiac surgery programs are often tertiary referral centres with high volume catheter laboratories and experienced staff. Our 30-year experience showed that even without on-site cardiac surgery, a high-volume standalone PCI centre can achieve excellent outcomes comparable with major centres in the world. The presence of on-site cardiac surgery is perhaps just a surrogate for an experienced high-volume PCI centre. Current trends suggest that future needs in coronary revascularization in the 21 st century in both developed as well as developing countries will in large part be fulfilled by standalone PCI centres.

FUNDING: This study is supported by a research grant from the Royal Alexandra Hospital Foundation.

DISCLOSURES: The authors have no additional financial disclosures or conflicts of interest to declare. guidelines) - executive summary: a report of the American College of Cardiology/American Heart Association Task Force on Practice Guidelines (committee to revise the 1993 guidelines for percutaneous transluminal coronary angioplasty). J Am Coll Cardiol 2001;37:2215-39.

12. Smith SC Jr, Feldman TE, Hirshfield JW Jr, et al. ACC/AHA/ SCAI 2005 guideline update for percutaneous coronary intervention - summary article: A report of the American College of Cardiology/ American Heart Association Task Force on Practice Guidelines (ACC/AHA/SCAI Writing Committee to Update the 2001 Guidelines for Percutaneous Coronary Intervention). J Am Coll Cardiol 2006;47:216-35.

13. Brown DC, Mogelson S, Harris R, Kemp D. Percutaneous coronary interventions in a rural hospital without surgical backup: Report of one year of experience. Clin Cardiol 2006;29:337-40.

14. Zavala-Alarcon E, Cecena F, Ashar R, Patel R, Van Poppel S, Carlson R. Safety of elective - including "high-risk" percutaneous coronary interventions without on-site cardiac surgery. Am Heart J 2004;148:676-83.

15. Paraschos A, Callwood D, Wightman MB, et al. Outcomes following elective percutaneous coronary intervention without on- site surgical backup in a community hospital. Am J Cardiol 2005;95:1091-3.

16. Frutkin AD, Mehta SK, Patel T, et al. Outcomes of 1,090 consecutive, elective, nonselected percutaneous coronary interventions at a community hospital without onsite cardiac surgery. Am J Cardiol 2008;101:53-7.

17. Turgeman Y, Atar S, Suleiman K, et al. Diagnostic and therapeutic percutaneous cardiac interventions without on-site surgical back-up - review of 11 years experience. Isr Med Assoc J 2003;5:89-93.

18. Melberg T, Nilsen DWT, Larsen AI et al. Nonemergent coronary angioplasty without on-site surgical backup: A randomized study evaluating outcomes in low-risk patients. Am Heart J 2006;5:888-95.

19. Carlsson J, James S, Stahle E, Hofer S, Lagerqvist B. Outcome of percutaneous coronary intervention in hospitals with and without on-site cardiac surgery standby. Heart 2007;93:335-8.

20. Herman BA, Iyer RN, Godier KJ. Safety and efficacy of offsite percutaneous coronary interventions in 1,348 consecutive patients in rural Tasmania. Am J Cardiol 2008;102:1323-7.

21. Glenn N. Levine, Eric R. Bates, James C. Blankenship, et al. 2011 ACCF/AHA/SCAI Guideline for Percutaneous Coronary Intervention: A Report of the American College of Cardiology Foundation/American Heart Association Task Force on Practice 
Guidelines and the Society for Cardiovascular Angiography and Interventions. J Am Coll Cardiol 2011;58:e44-122.

22. Zia MI, Wijeysundera HC, Tu JV, Lee DS, Ko DT. Percutaneous coronary interention with vs without on-site cardiac surgery backup: A systematic review and meta-analysis. Can J Cardiol 2011:664:e9-e16.

23. Aversano T, Lemmon CC, Liu L et al. Outcomes of PCI at hospitals with of without on-site cardiac surgery. N Engl J Med 2012;366:1792-802.

24. Jacobs A, Normand S, Massaro J et al. Nonemergency PCI at hospitals with or without on-site cardiac surgery. N Engl J Med 2013;368:1498-508.

25. Klinke WP, Hui W. Percutaneous transluminal coronary angioplasty without on-site surgical facilities. Am J Cardiol 1992;70:1520-5.

26. Klinke WP, Kubac G, Talibi T, Lee SJK. Safety of outpatient cardiac catheterizations. Am J Cardiol 1985;56:639-41.

27. APPROACH - Alberta Provincial Project for Outcomes Assessment in Coronary Heart Disease. <www.approach.org> (Accessed December 28, 2014)

28. Gruntzig AR, Senning A, Siegenthaler WE. Nonoperative dilatation of coronary-artery stenosis: Percutaneous transluminal coronary angioplasty. N Engl J Med 1979;301:61-8.

29. Kent KM. Interventional cardiology: 1990s (Editorial). Am J Cardiol 1992;70:1607-8.

30. Loop FD, Whitlow PL. "Beauty is in the Eye..." (Editorial). Am J Cardiol 1992;70:1608-9.

31. Loubeyre C, Morice MC, Berzin B, et al. Emergency coronary artery bypass surgery following coronary angioplasty and stenting: Results of a French multicenter registry. Catheter Cardiovasc Interv 1999;47:441-8.

32. Seshadri N, Whitlow PL, Acharya N, Houghtaling P, Blackstone EH, Ellis SG. Emergency coronary artery bypass surgery in the contemporary percutaneous intervention era. Circulation 2002;106:2346-50.

33. Yang EH, Gumina RJ, Lennon RJ, Holmes DR, Rihal CS, Singh M. Emergency coronary artery bypass surgery for PCIs: Change in the incidence, clinical characteristics, and indications 1979-2003. J Am Coll Cardiol 2005;46:2004-9.

34. National Audit of Percutaneous Coronary Interventional Procedures Annual Report 2011. British Cardiovascular Intervention Society $<$ www.bcis.org.uk> (Accessed December 28, 2014).
35. Kutcher MA, Klein LW, Ou F-S et al. Percutaneous coronary interventions in facilities without cardiac surgery on site: A report from the National Cardiovascular Data Registry (NCDR). J Am Coll Cardiol 2009;54:15-24.

36. McGrath PD, Wennberg DE, Malenka DJ et al. Operator volume and outcomes in 12,988 percutaneous coronary interventions. J Am Coll Cardiol 1998;31:570-6.

37. Hannan EL, Wu C, Walford G, et al. Volume outcome relationships for percutaneous coronary interventions in the stent era. Circulation 2005;112:1171-9.

38. Srinivas VS, Hailpern SM, Koss E, Monrad ES, Alderman MH. Effect of physician volume on the relationship between hospital volume and mortality during primary angioplasty. J Am Coll Cardiol 2009;53:574-9.

39. Dawkins KD, Gershlick T, de Belder M, et al. Coronary angioplasty: Guidelines for good practice and training. Heart 2005;91(Suppl 6):vi1-vi27.

40. Burton KR, Slack R, Oldroyd KG, et al. Hospital volume of throughput and periprocedural and medium-term adverse events after percutaneous coronary intervention: retrospective cohort study of all 17,417 procedures undertaken in Scotland, 1997-2003. Heart 2006;92:1667-72.

41. British Heart Foundation Statistics Database Coronary Heart Disease Statistics 2010 Table 3.2. <www.heartstats.org> (Accessed December 28, 2014)

42. Epstein AJ, Polsky D, Yang F, Yang L, Groeneveld PW. Coronary revascularization trends in the United States, 2001-2008. JAMA 2011;305:1769-76.

43. McMurtry MS, Southern DA, Lewin AM, et al. Recent temporal trends and geographic distribution of cardiac procedures in Alberta. Can J Cardiol 2013;29:460-5.

44. Cardiac Care Network of Ontario. Stand-alone Angioplasty External Evaluation - Final Report \& Recommendations. <www.ccn.on.ca/> (Accessed October 4, 2015).

45. Patel AN, Tu JV, Waters NM, et al. Access to primary percutaneous coronary intervention for ST-segment elevation myocardial infarction in Canada: A geographic analysis. Open Med 2010;4:1. 